\title{
The model of magnetic-field-controlled shape memory effect in NiMnGa
}

\author{
A.A. Likhachev and K. Ullakko ${ }^{1}$ \\ Institute of Metal Physics, Department of Phase Transitions, Vernadsky St. 36, 252142 Kiev, \\ Ukraine \\ ${ }^{1}$ Helsinki University of Technology, Laboratory of Engineering Physics and Mathematics, \\ Rakentajankio 2C, 02150 Espoo, Finland
}

\begin{abstract}
This report represents some new experimental results and the quantitative model describing large magneto-strain effect and main mechanical and magnetic properties observed in several ferromagnetic shapememory alloys. The model application to giant magneto-strain effect recently found in some non-stoichiometric Ni$\mathrm{Mn}-\mathrm{Ga}$ alloys is discussed.
\end{abstract}

\section{INTRODUCTION}

Some ferromagnetic shape memory alloys were recently suggested as a general way for the development of a new class of the magnetic-field-controlled actuator materials that will allow control of large strain effect by application of a magnetic field. Numerous candidate shape memory materials were explored including $\mathrm{Ni}_{2} \mathrm{MnGa} \mathrm{Co}_{2} \mathrm{MnGa}$, FePt CoNi, and FeNiCoTi during past few years. Large magnetically driven strain effects are expected have to occur in these systems. Up to date, the largest magneto-strain effects were achieved in Ni-Mn-Ga ferromagnetic shape memory alloys [1-7]. The best results on the field-induced strain reported short time ago [2] never exceeded $0.2-0.3 \%$ strain value in a magnetic field of order $8 \mathrm{kOe}$ in martensitic state for nearly stoichiometric single-crystal samples of $\mathrm{Ni}_{2} \mathrm{MnGa}$ alloy. Recently, several research groups [6,7] have reported on the observation of super-large more than 5\% magneto-strain effect in some non-stoichiometric Ni-Mn-Ga alloys close to that $5.78 \%$ value expected from the tetragonality aspect ratio of the martensite crystal lattice. New Ni-Mn-Ga alloys showing giant magneto-strain effect display simultaneously few interesting physical effects and new behavior for some magnetic and mechanical properties that is very different from that earlier observed in $\mathrm{Ni}_{2} \mathrm{MnGa}$ with lower $(<0.31 \%)$ magneto-strain effect.

One the new non-stoichiometric $\mathrm{Ni}_{48} \mathrm{Mn}_{30} \mathrm{Ga}_{22}$ alloy has recently been studied in details. This alloy undergoes the martensitic transformation at about $35 \mathrm{C}^{0}$ from the $\mathrm{L} 21$ ordered Heusler type cubic $(\mathrm{a}=$ $0.582 \mathrm{~nm}$ ) crystal structure into a tetragonally distorted structure with crystalline lattice parameters: $\mathrm{a}=\mathrm{b}=0.594 \mathrm{~nm}$ and $\mathrm{c}=0.562 \mathrm{~nm}$. The martensitic phase is ferromagnetic one and internally twinned. It contains a mixture of twin variants twinned usually on $\{110\}$ planes. The orientation of their c-axes is nearly parallel to [100] directions of the parent cubic phase. Tetragonal symmetry direction is simultaneously a single easy magnetization axis for each martensitic variant. Very low 2-3 MPa compressive twinning stress along [100] directions needed to transform the multi-variant samples into a single-variant state has been found for $\mathrm{Ni}-\mathrm{Mn}-\mathrm{Ga}$ alloys displaying giant field-induced deformation effect. It is much less than it was observed in some earlier studied alloys (15-20 MPa [8-9]).

As a result, new alloys that usually have very simple two-variant twinning microstructure and very low twinning stresses can be easily transformed between two single twin variants by application both the mechanical stress and the magnetic field through the mechanism of twin boundaries motion.

Simultaneously, some important steps were made during the past few years concerning the physical nature of the magneto-mechanical phenomena observed in ferromagnetic shape memory alloys. James and Wuttig [4] developed the models based on a constrained magnetization orientation approach. O'Handley [5] first included the rotation magnetization effects together with usual Zeeman energy term 
into consideration. Likhachev and Ullakko [3] have developed a general thermodynamic approach based on the Maxwell's relationships and derived the main magneto-mechanical equation representing both the magnetic and the mechanical driving force balance in ferromagnetic shape memory materials. The last model has been also successfully applied to explain quantitatively the results of experimental study of large magneto-strain effects in $\mathrm{Ni}_{2} \mathrm{MnGa}$ earlier obtained in [2]. In particular, according to our model estimation made in [3] a giant magneto-strain value can be achieved only in materials with very low $(<2$ $\mathrm{MPa}$ ) twinning stress values. Mechanical testing results obtained for giant magneto-strain $\mathrm{Ni}-\mathrm{Mn}-\mathrm{Ga}$ samples have completely confirmed these estimations. The present report represents some experimental results and several new effects recently found in some non-stoichiometric Ni-Mn-Ga alloys that are directly connected with their giant magneto-strain response. We also represent some model background based on the magnetization free energy consideration that allows better understand its important role. Naturally, this approach gives the same final equations derived in our preceding publications. Finally, the model equations are applied to explain some new experimental results.

\section{MAGNETIC DRIVING FORCES AND TWIN BOUNDARY MOTION}

Perhaps, starting from a simplest physical idea first clearly formulated in [1], it is generally believed that a large macroscopic mechanical strain induced by the magnetic field in some ferromagnetic shape memory alloys is usually realized trough the twin boundaries motion and redistribution of different twin variant fractions in a magnetic field. In this case, the main thermodynamic driving forces should have a magnetic nature and be connected with high magnetization anisotropy and significant differences in magnetization free energies for different twin variants of martensite [1-7].

For instance, Fig.1 shows an example of giant 5\% magneto-strain effect associated with the direct and reverse magnetic-field-induced transformation between two single twin variants in $\mathrm{Ni}_{48} \mathrm{Mn}_{30} \mathrm{Ga}_{22}$ sample. The magneto-strain value achieved in this new alloy is insufficiently less compared to the natural crystallographic limit $1-\mathrm{c} / \mathrm{a}=5.78 \%$ expected for twinning in this material.

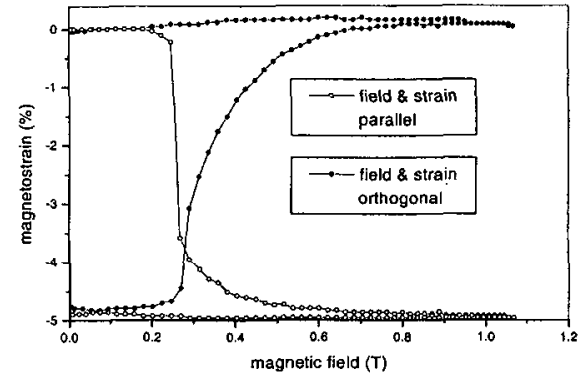

Fig.1. Giant compression and extension magnetostrain effects induced by magnetic field in $\mathrm{Ni}_{48} \mathbf{M n}_{30} \mathrm{Ga}_{22}$ alloy.

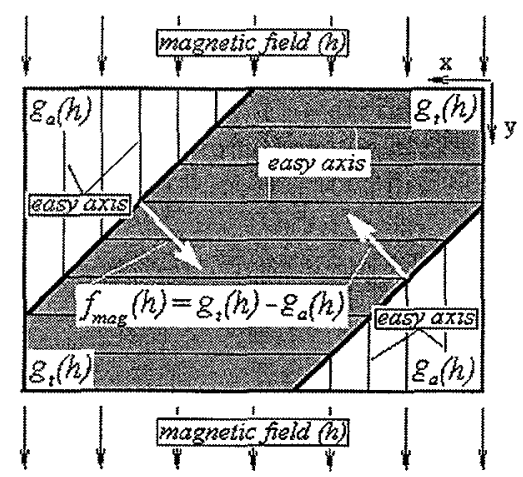

Fig.2. Two-variant twin microstructure, easy magnetization axes alignment and magnetic driving force responsible for twin boundary motion in a magnetic field applied.

Fig.2 schematically shows a typical for some new Ni-Mn-Ga alloys two-variant twin microstructure, orientation of easy and hard magnetization axes for both twin variants and also the magnetic driving force alignment that usually give the maximal value of the magnetic field-induced strain in this material.

It should be noted that some early models have considered each twin band as a single magnetic domain with a constant magnetization value and constrained magnetization direction. This approach predicted some unlimited linear growth of the magnetic driving force in a magnetic field applied. Respectively, it was expected that one can easily obtain significant magnetostrain effects in ferromagnetic shape memory 
alloys trough the twin boundary motion simply increasing magnetic field value as high as neded. It has been understood later $[5,3]$ that it is not so, because each twin band is not a single magnetic domain but should contain the multiple internal magnetic domain microstructure and typical expected for the uniaxial ferromagnets $180^{\circ}$ domain walls. As a result, the local average magnetization inside of twins is not a constant and can be canged in a magnetic field due to both the magnetic domain wall motion and the rotation of magnetization. The most important conclusion following from this point of view is that the magnetic driving force can not be increased infinitely in a magnetic field but always limited its maximal value independent on the magnetic field and proportional the magnetic anisotropy constant. This value is low enough, so only the very soft (in sense of twinning stress) materials can be good candidates to show really high magnetic field induced strain effects. Therefore, the investigation of magnetic properties is the first important task for the magnetic driving force calculation and understanding of the magnetomechanical behavior of $\mathrm{Ni}-\mathrm{Mn}-\mathrm{Ga}$ and similar type alloys.

Some results showing main magnetic properties of $\mathrm{Ni}_{48} \mathrm{Mn}_{30} \mathrm{Ga}_{22}$ in martensitic state are indicated in Fig.3. First, the magnetization behavior of a single variant of martensitic phase has been studied. For this aim the internally twinned sample was initially transformed into a single variant state by application of compressive stress $>3 \mathrm{MPa}$ and then hardly constrained without unloading in a special holder. Two magnetization loops displaying field dependence of the magnetization $m_{a}(h)$ along the easy (tetragonal symmetry) axis and also along the hard magnetization directions $m_{t}(h)$ were measured. Corresponding magnetization free energies can be calculated from these magnetization data as functions of the magnetic field applied as follows:

$$
g_{a}(h)=-\int_{0}^{h} m_{a}(h) d h \quad \text { and } \quad g_{i}(h)=-\int_{0}^{h} m_{i}(h) d h
$$

Different mechanisms of magnetization for easy and hard magnetization directions are expected, respectively. In particular, $180^{\circ}$ magnetic domain walls motion is expected to be the main magnetization mechanism for easy axis. So as, the rotation of magnetization without domain walls motion seems to be responsible for the magnetization behavior in a hard direction [5].

These results are shown in Fig.3. The energy of magnetic anisotropy defined as a difference of these free energies is also indicated on this plot. In particular, it can be easily found that the magnetic driving force responsible for twin boundaries motion is practically equal to the magnetic anisotropy energy. For this aim one should also know the magnetization free energy in the multi-variant sate. Its dependence on the relative volume fractions of different martensite variants is especially important.

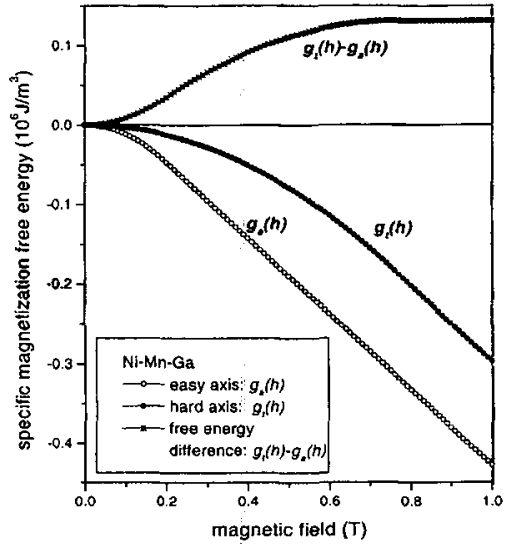

Fig.3. Magnetic anisotropy of $\mathrm{Ni}_{48} \mathrm{Mn}_{30} \mathrm{Ga}_{22}$ and field dependence of magnetization free energies for easy and hard magnetization directions

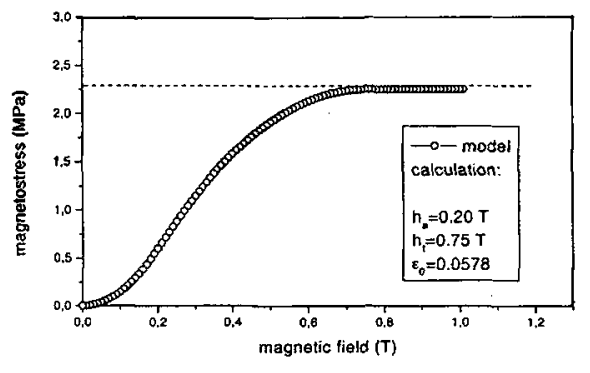

Fig.4. Mechanical stress induced by magnetic field obtained from the model calculation. 
In case of simple two-variant twinning geometry shown in Fig. 2 the easy magnetization axis of the first variant (white area) is parallel to a magnetic field applied. For the second one (gray color) the magnetic field is applied in a transversal hard magnetization direction. Further, both these variants are called axial (a) and transversal $(t)$, respectively. Taking the specific magnetization free energies for both twin variants $g_{a}(h)$ and $g_{t}(h)$ found, for instance, from the experiment (see Fig.3) one can write the total magnetization free energy per unit volume of the material as folows:

$$
g_{\text {mag }}(h, x)=x g_{a}(h)+(1-x) g_{t}(h)=g_{t}(h)+x\left(g_{a}(h)-g_{t}(h)\right)
$$

Therefore, due to magnetic anisotropy the magnetization free energy becomes dependent on the relative volume fractions $x$ and 1 - $x$ occupied by axial and transversal twin variants, correspondingly. According to our previous calculation of the magnetization free energies shown in Fig. $3 g_{a}(h)<g_{l}(h)$ So, one can decrease $g_{\text {mag }}(h, x)$ from its maximal value $g_{d}(h)$ at $x=0$ to the minimal one $g_{a}(h)$ at $x=1$ moving the twin boundaries as indicated in Fig.2. The corresponding magnetic driving force moving twin boundaries along their normal directions can be found from the general thermodynamic rule as follows:

$$
f_{\text {mag }}(h)=-\left[\frac{\partial}{\partial x} g_{\text {mag }}(h, x)\right]_{h}=-\left(g_{a}(h)-g_{t}(h)\right)
$$

Therefore, a non-zero magnetic driving force responsible for twin boundary motion appears as a result of uniaxial magnetic anisotropy of $\mathrm{Ni}-\mathrm{Mn}-\mathrm{Ga}$. This force is applied along the twin boundary normal direction and equal to a difference in magnetization free energies between the different twin variants. The magnetic driving force is dependent on the magnetic field applied and never can exceed its saturation level as shown in Fig.3. The maximal estimated value about of $0.13^{*} 10^{6} \mathrm{~N} / \mathrm{m}^{2}$ is achieved at $h>0.8 \mathrm{~T}$ for $\mathrm{NiMnGa}$ alloy.

\section{MAGNETO-MECHANICAL BALANCE EQUATION AND MAGNETO-STRAIN EFFECT}

According our model approach [3] the mechanical effect of the magnetic field applied can be always represented through some equivalent mechanical stress. This magnetic-field-induced stress $\sigma_{\text {mag }}(h)$ can be always found from the following general equation:

$$
\sigma_{\text {mas }}(h)=\varepsilon_{0}^{-1} \int_{0}^{h}\left(m_{a}(h)-m_{t}(h)\right) d h=\varepsilon_{0}^{-1}\left(g_{t}(h)-g_{a}(h)\right)
$$

Therefore, the equivavalent magnetic stress is proportional to the magnetic driving force applied and reverce proportional to twinning strain value $\varepsilon_{0}=1$-c/a. This equivalent magnetic stress $\sigma_{\text {mag }}(h)$ can be easily calculated from the Eq.(4). For this aim one needs only to know the magnetization properties of the material and use the magnetic driving force calculation data indicated in Fig.3. According to our calculations shown in Fig. 4 the maximal equivalent magnetic stress that can be developed in a magnetic field is at about $2.25 \mathrm{MPa}$ in $\mathrm{Ni}_{48} \mathrm{Mn}_{30} \mathrm{Ga}_{22}$.

On the other side, according to results of the mechanical testing experiments shown in Fig.5 the uniaxial mechanical stress required to transform completely $\mathrm{Ni}_{48} \mathrm{Mn}_{30} \mathrm{Ga}_{22}$ samples from one single variant of martensitic phase to another one through the stress-induced twin boundaries motion does not exceed 2.5 $\mathrm{MPa}$. The maximal compression strain that can be achieved on this load is about $5.8 \%$. Therefore, due to very low twinning stress value recently found in some $\mathrm{Ni}-\mathrm{Mn}-\mathrm{Ga}$ alloys the calculated magnetic stress is completely enough to achieve very high $5 \%$ magneto-strain value in these new materials.

All the forces including the external compressive stress $\sigma$, magnetic field induced stress $\sigma_{\text {mag }}(h)$ and the opposite internal mechanical stress $\sigma_{\text {mech }}(\varepsilon)$ should be in total balance. So, one should write a general force balance equation as follows:

$$
\sigma_{\text {mech }}(\varepsilon)=\sigma+\sigma_{\text {mag }}(h)
$$

Both these general expressions have been derived in [3] directly from the thermodynamic Maxwell's rules. 
At zero magnetic field $\sigma_{\text {mag }}(0)=0$ and Eq.(5) is reduced to usual mechanical stress-strain relationship $\sigma=\sigma_{\text {mech }}(\varepsilon)$. So, $\sigma_{\text {mech }}(\varepsilon)$ can be always obtained from the mechanical testing data. Using the reverse function $\varepsilon=\varepsilon_{\text {mech }}(\sigma)$ shown in Fig.5 one can obtain the general solution of the main force balance equation (5) in respect to $\mathcal{E}$ as folows:

$$
\varepsilon^{m s m}(h)=\varepsilon_{\text {mech }}\left(\sigma_{\text {mag }}(h)\right), \text { at } \sigma=0
$$

\section{MODEL CALCULATIONS}

Some calculation results that follows from our model consideration are presented in this section. In particular, Fig. 5 represents both the strain-stress hysteresis loop found from the experiment and also a set of model fitting curves corresponding to different values of twinning stress. Simple Fermi-like distribution functions were used as an appropriate fitting basis for analytic interpolation of the mechanical testing results.

$$
\varepsilon_{ \pm}^{\text {mec }}(\sigma)=\varepsilon_{0}\left(1+\exp \frac{ \pm \sigma_{0}-\sigma}{\Delta \sigma}\right)^{-1}
$$

Here, \pm denote loading and unloading curves respectively. $\sigma_{0}$ and $\Delta \sigma$ are characteristic stress parameters that can be associated with the start $\sigma_{s}=\sigma_{0}-2 \Delta \sigma$ and finish $\sigma_{f}=\sigma_{0}+2 \Delta \sigma$ twinning stress values. The best fit between the model function and experimental data is achieved at $\sigma_{0}=1.56 \mathrm{MPa}$ and $\Delta \sigma=0.26 \mathrm{MPa}$ as indicated in Fig. 5 by the solid circle labelled line. Additional mechanical hysteresis loops $(a-b, c-e)$ shown here were also simulated to study the effect of different twinning stress values on the magneto-strain behavior. The corresponding model calculation results performed in accordance with Eq.(6) and the experimental measurement data for the magnetic-field-controlled strain behavior in $\mathrm{Ni}_{48} \mathrm{Mn}_{30} \mathrm{Ga}_{22}$ are plotted altogether in Fig.6. As follows from these calculations the increase of twinning stress may cause the gradual decrease of the magnetic field induced strain from its maximal possible value $5.8 \%$ at $\sigma_{f}<1.67 \mathrm{MPa}$ to $2 \%$ at $\sigma_{f}=3.32 \mathrm{MPa}$. In particular, one can observe the reasonable quantitative agreement between the $5.5 \%$ value expected from the model calculation and $5.2 \%$ experimental one.

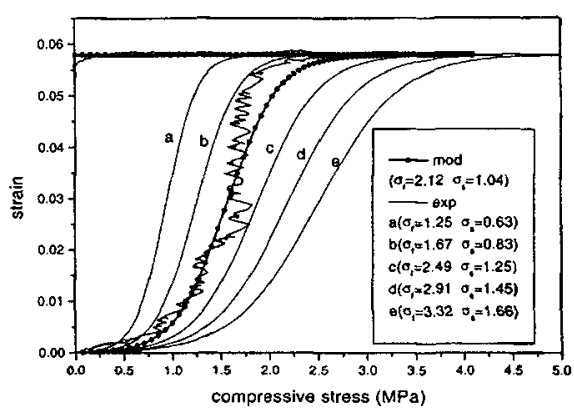

Fig.5. Strain-stress behavior caused by transformation between two single twin variants induced by mechanical stress in Ni-Mn-Ga martensite.

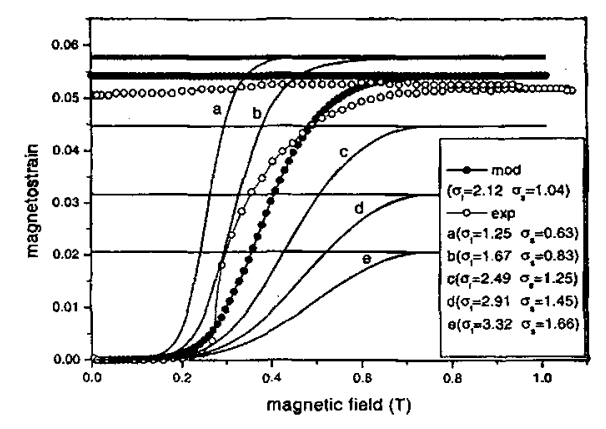

Fig.6. Model calculation result of the magnetic field induced strain effect in comparison with experiment.

Therefore, one can conclude that the low twinning stress value is a very critical physical parameter controlling a super-large magneto-strain effect in NiMnGa. In particular, this can explain considerably lower magneto-strain effect found earlier in some nearly stoichiometric alloys which had at almost ten 
times higher (15-20MPa) twinning stresses. So, the magnetic driving forces (that can produce no more than 2-3MPa equivalent stress) were only able insufficiently redistribute the relative twin variant fractions in these materials. As a result no more than $0.2 \%$ deformation effect was observed.

\section{CONCLUSIONS}

1. A super-large deformation effect at about $5 \%$ that can be induced by application of magnetic field less than $1 \mathrm{~T}$ in some new ferromagnetic non-stoichiometric NiMnGa shape memory alloys seems to be the most interesting property of this material.

2. NiMnGa alloys displaying giant field-induced deformation effects have always very simple practically two-variant twin microstructure. As a result, these alloys can be easily deformed by application of the low $<2 \mathrm{MPa}$ mechanical stress or magnetic field less $0.8 \mathrm{~T}$ through the mechanism of twin boundary motion. In particular, the reversible magneto-mechanical transformation cycle between two twin variants of martensitic phase can be induced by subsequent application of magnetic field along two different perpendicular directions.

3. The magnetic driving force applied to twin boundaries is equal to a difference in magnetization free energies between the different twin variants of martensite. This difference also characterizes the energy of uniaxial magnetization anisotropy and can be calculated from the corresponding magnetization measurements. The magnetic driving force achieves its maximal value $0.13 \mathrm{MN} / \mathrm{m}^{2}$ in a magnetic field higher $0.8 \mathrm{~T}$. In agreement with our earlier estimations and present model calculations this value is completely enough to explain high $5 \%$ magnetic field induced strain in some $\mathrm{NiMnGa}$ alloys which have the very low $<2 \mathrm{MPa}$ twinning stress.

4. There is a definite analogy between the deformation effects caused by the mechanical and magnetic driving forces. For instance, in both cases the macroscopic twining strain driven by the mechanical stress or the magnetic field applied can be expressed through the same universal function dependent on the corresponding (mechanical or magnetic) driving force.

\section{References}

1. K. Ullakko J. Met. Eng. Perform., 5, 405 (1996).

2. Ullakko, J. K. Huang, C. Kantner, R. C. O'Handley and V. V. Kokorin, Appl. Phys. Lett., 9, 1966 (1996).

3. A.A. Likhachev and K. Ullakko, EPJdir, B2, pp. 1-9 (1999); Eur. Phys. J, B14, 263 (2000).

4. R. D. James and M. Wuttig, Philos. Mag. A 77, 1273 (1988).

5. R.C. O'Handley, J. Appl. Phys., 83, 3263 (1998).

6. K. Ullakko, A.Sozinov, and P.Yakovenko, E-print, ArXiv.org/cond-mat/0004211 (2000).

7. S.J. Murrey, R. Hayashi, M. Marioni, S.M. Allen, R.C. O'Handley., In Smart Structures and Materials 1999: Smart Materials Technologies, ed. Manfred Wuttig, USA. 1999, SPIE3675, pp. 204211.

8. V. V. Kokorin and V. V. Martinov Fiz. Met. Metalloved., 9, 106 (1991).

9. V. V. Martinov and V. V. Kokorin J. Phys. (France) III 2, 739 (1992). 